\title{
Nintedanib in IPF: Post hoc Analysis of the Italian FIBRONET Observational Study
}

\author{
Sergio Hararia ${ }^{a} b$ Alberto Pesci ${ }^{c}$ Carlo Albera $^{d}$ Venerino Poletti ${ }^{\mathrm{e}}{ }^{\mathrm{f}}$ \\ Christian Amici ${ }^{g}$ Giovanna Crespi ${ }^{\text {h }}$ Benedetta Campolo ${ }^{\text {h }}$ Carlo Vancheri ${ }^{i}$ \\ on behalf of the FIBRONET study group
}

\begin{abstract}
aDepartment of Clinical Sciences and Community Health, University of Milan, Milan, Italy; ${ }^{b}$ U.O. di Pneumologia e Terapia Semi-Intensiva Respiratoria, Ospedale San Giuseppe, IRCCS MultiMedica, Milan, Italy; ${ }^{\mathrm{C} D e p a r t m e n t}$ of Medicine, Respiratory Unit, University of Milano-Bicocca, ASST Monza, Monza, Italy; ${ }^{d}$ SC Pneumologia U, A.O.U. Città Della Scienza e della Salute (Molinette), University of Torino, Turin, Italy; ${ }^{\text {} D e p a r t m e n t ~ o f ~ D i s e a s e s ~ o f ~ t h e ~ T h o r a x, ~}$

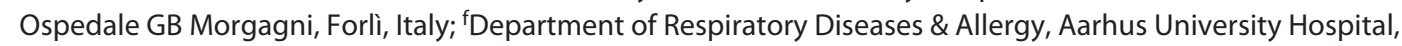

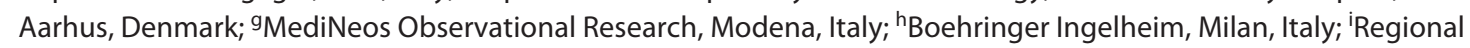
Referral Centre for Rare Lung Diseases, University-Hospital “Policlinico G. Rodolico”, Department of Clinical and Experimental Medicine, University of Catania, Catania, Italy
\end{abstract}

\section{Keywords}

Idiopathic pulmonary fibrosis · Nintedanib · Antifibrotic treatment · Observational study · Lung function

\begin{abstract}
Background: The FIBRONET study was an observational study of patients with idiopathic pulmonary fibrosis (IPF) in Italy. Objectives: In this post hoc descriptive analysis, we describe changes in lung function, anxiety/depression, coughing, exacerbations, and adverse events (AEs) in patients receiving nintedanib treatment. Methods: Patients with IPF from 20 centers in Italy, aged $\geq 40$ years who received nintedanib for $\geq 7$ months, were followed up for 12 months from study enrollment, attending clinic visits every 3 months. Outcomes included change in forced vital capacity (FVC)\% predicted from baseline to 12 months, anxiety/depression measured by the Hospital Anxiety and Depression Scale (HADS), and the proportion of patients with cough, AEs, and exacerbations. Results: In total, 52 patients received nintedanib (mean duration of 11.6 months). Ten patients had dose re-
\end{abstract}

ductions from $150 \mathrm{mg}$ to $100 \mathrm{mg}$ twice daily, due to AEs. FVC\% predicted was unchanged in the overall nintedanib population (78.7\% at baseline; $79.8 \%$ at 12 months) and those with a reduced dose $(77.7 \%$ at baseline; $81.0 \%$ at 12 months). HADS score was low at baseline and throughout the study. The proportion of patients with cough decreased from $50.0 \%$ to $21.2 \%$ over 12 months. Two patients experienced exacerbations, 2 patients discontinued treatment, and 27 (51.9\%) reported AEs. The most common AE was diarrhea (34.6\%). Conclusions: In patients with IPF who received nintedanib in the FIBRONET study, FVC\% predicted was stable over 12 months, and the proportion of patients with cough decreased. The safety profile was consistent with the known safety profile for nintedanib in IPF.

\section{(c) 2022 The Author(s)}

Published by S. Karger AG, Basel

Benedetta Campolo was an employee of Boehringer Ingelheim (Italy) at the time of this study.

Trial registration: This study was registered at clinicaltrials.gov; https://clinicaltrials.gov/ct2/show/NCT02803580.
(C) 2022 The Author(s).

Published by S. Karger AG, Basel

This is an Open Access article licensed under the Creative Commons Attribution-NonCommercial-4.0 International License (CC BY-NC) (http://www.karger.com/Services/OpenAccessLicense), applicable to the online version of the article only. Usage and distribution for commercial purposes requires written permission. 


\section{Introduction}

Nintedanib is a tyrosine kinase inhibitor that was approved for the treatment of idiopathic pulmonary fibrosis (IPF) in the USA in 2014 and in the EU in $2015[1,2]$ based on the results of two replicate, phase III clinical trials (INPULSIS- 1 and INPULSIS-2). In these trials, nintedanib significantly slowed the annual rate of decline in percent predicted forced vital capacity (FVC\% predicted) versus placebo during 52 weeks of treatment [3]. A higher proportion of patients treated with nintedanib compared with placebo had no decline in $\mathrm{FVC} \%$ predicted, and a smaller proportion had an absolute decline in $\mathrm{FVC} \%$ predicted $\geq 5 \%$ or $\geq 10 \%$ from baseline to week 52 , indicating greater preservation of lung function in patients receiving nintedanib [4].

Since the regulatory approval of nintedanib, a growing body of real-world evidence has emerged from various registry and other real-world studies conducted in countries in Europe [5-11], as well as in the USA [12] and Australia [13]. Data from these real-world, observational studies support the findings of the nintedanib clinical trial program and show nintedanib to be effective for the management of IPF in terms of reducing disease progression. The real-world evidence also supports the clinical trial finding that gastrointestinal events, particularly diarrhea, are the most frequent adverse events (AEs) caused by nintedanib [5].

The FIBRONET study (registered at clinicaltrials.gov; NCT02803580) was a real-world study conducted in Italy to describe the clinical characteristics and course of IPF and the use of antifibrotic medications [14]. The mean FVC\% predicted was $80.0 \%$ at baseline and $82.2 \%$ at 12 months, and $47.4 \%$ of patients remained stable (i.e., had no disease progression) in terms of $\mathrm{FVC} \%$ predicted during the study. The mean time between diagnosis and initiation of antifibrotic treatment was 6.4 weeks.

In this post hoc descriptive analysis of the FIBRONET study, we analyzed patients who received nintedanib for $\geq 7$ months during the 12 -month observation period. Our aims were to investigate the effectiveness and safety of nintedanib in patients with IPF by describing changes in lung function, levels of anxiety/depression, AEs and serious AEs (SAEs), coughing, and exacerbations in a realworld setting.

\section{Materials and Methods}

The FIBRONET study was a 12-month, observational, prospective cohort study of 209 patients in Italy aged $\geq 40$ years and diagnosed with IPF in the 3 months prior to enrollment, according to 2011 American Thoracic Society/European Respiratory Society/
Japanese Respiratory Society/Latin American Thoracic Society guidelines [15] (the study was conducted before the 2018 update). Diagnosis of IPF was at the participating center and was not centrally reviewed, as this was an observational study conducted according to clinical practice. After enrollment, patients were followed up for 1 year, attending three follow-up evaluations at 3 $(+/-1.5), 6(+/-1.5)$, and $9(+/-1.5)$ months (in accordance with current clinical practice in Italy for the management of patients with IPF), plus a final evaluation at $12(+/-1.5)$ months. In total, 20 pulmonary centers, responsible for managing the majority of patients with IPF in Italy, were involved in the study. The full design of the FIBRONET study, including inclusion/exclusion criteria, has been published previously. The protocol for the FIBRONET study was approved in July 2015 by an institutional review board/ethics committee (107/2015/PO del registry EC), and written informed consent was obtained for all patients.

Patients were eligible for inclusion in this post hoc analysis if they had received treatment with nintedanib for $\geq 7$ months during the 12 months of observation and had available FVC\% predicted data at baseline and at 12-month follow-up. This was used to allow for a relatively homogeneous sample of patients with regard to nintedanib treatment, based on the distribution of exposure times in the study population.

\section{Outcomes}

In this post hoc analysis, outcomes included the proportion of patients with preserved lung function (defined as FVC predicted $\geq 80 \%$ ) at baseline [16], the change in FVC\% predicted over time, and the proportion of patients with a decline in $\mathrm{FVC} \%$ predicted. These endpoints were assessed in all patients who met the criteria for the post hoc analysis, and the subset who had their dose reduced to $100 \mathrm{mg}$ twice daily (BID) during the observational period.

In addition, levels of anxiety and depression were evaluated at baseline (at enrollment) and at 6- and 12-month follow-up visits, using the Hospital Anxiety and Depression Scale (HADS), a commonly used, 14-item scale used to determine levels of anxiety and depression. On the HADS, seven items relate to anxiety and seven relate to depression. Each question on the scale is scored 0-3, meaning that the maximum score for either anxiety or depression is 21 , and the maximum total score is 42 .

We also investigated the proportion of patients with "cough" symptoms (evaluated by the clinician during study visits using clinical judgment) at baseline and 3-, 6-, 9-, and 12-month followup. We assessed the pattern of cough in patients based on the presence/absence of cough between study visits. Investigators recorded AEs and SAEs between IPF diagnosis and end of the observation period, and all adverse drug reactions and fatal AEs were collected in the electronic case report form. The proportions of patients with acute exacerbations during the observation period were also recorded. An acute exacerbation was defined as a sudden acceleration of the disease or an idiopathic acute injury superimposed on the diseased lung that led to a significant decline in lung function and was classified as mild, moderate, or severe according to the clinical judgment [17]. Finally, the dosage of nintedanib administered during the observation period was reported.

\section{Statistics}

Data were analyzed descriptively and are presented as means, medians, and proportions, according to the nature of the variables. Standard deviation (SD) and interquartile range were used as dis- 
persion measures. Missing FVC\% predicted data at 12 months were imputed using a Last Observation Carried Forward (LOCF) approach. The LOCF approach was applied to all patients with FVC\% predicted values available at baseline and at least one follow-up visit $(3,6$, or 9 months).

\section{Results}

\section{Patient Characteristics}

The analysis included 52 patients who received nintedanib for $\geq 7$ months and had evaluable data at baseline and 12 months; mean age was 70.2 years, and $78.8 \%$ of patients were male. Eight patients who received nintedanib and had data at baseline and 12 months were excluded due to $<7$ months of nintedanib treatment duration following treatment discontinuation $(n=3)$, prior use of pirfenidone before switching to nintedanib $(n=3)$, switching to pirfenidone $(n=1)$, and treatment interruption for 5 months $(n=$ $1)$. Mean time from first IPF diagnosis to enrollment was 0.9 months, and the majority of patients had at least one comorbidity (Table 1). Fifty-two patients had FVC\% predicted data available at baseline, 38 at 3 months, 43 at 6 months, 29 at 9 months, and 52 at 12 months. At baseline, 22 of the 52 evaluable patients $(42.3 \%)$ had preserved lung function ( $\geq 80 \%$ FVC predicted [16]), and mean diffusing capacity of the lungs for carbon monoxide $\left(\mathrm{DL}_{\mathrm{CO}}\right)$ percent predicted was 51.7 (Table 1).

The mean $( \pm S D)$ duration of nintedanib treatment was $11.6( \pm 1.6)$ months. Forty out of 52 patients $(76.9 \%)$ received $150 \mathrm{mg}$ BID nintedanib, and 10/52 patients (19.2\%) reduced the dose to $100 \mathrm{mg}$ BID during the observational period to manage AEs. Two patients who received nintedanib for at least 7 months discontinued nintedanib, due to SAEs.

\section{FVC\% Predicted}

At baseline, mean $( \pm \mathrm{SD}) \mathrm{FVC} \%$ predicted was $78.7 \%$ $( \pm 15.0)$ (Table 2). There was no change in FVC\% predicted over time; at follow-up, mean values were $79.8 \%$ $( \pm 14.6), 80.7 \%( \pm 17.2), 80.6 \%( \pm 18.2)$, and $79.8 \%( \pm 15.5)$ at $3,6,9$, and 12 months, respectively. Considering the relative change in $\mathrm{FVC} \%$ predicted during the 12 months of observation, $25.0 \%(13 / 52)$ of patients were classified as "decliners" (decline $\geq 5 \%$ ) [18, 19] (Fig. 1), with $15.3 \%$ $(8 / 52)$ of patients demonstrating a decline of $\geq 10 \%$. Seventy-five percent (39/52) of patients were classified as "nondecliners" (they had a decline $<5 \%$ or increase in FVC\% predicted), with $44.2 \%(23 / 52)$ of patients classified as “improvers" (>5\% increase in FVC) (Fig. 1).
Table 1. Patient characteristics at enrollment

\begin{tabular}{ll}
\hline Characteristic & Patients $(N=52)$ \\
\hline Mean age, years (SD) & $70.2(7.1)$ \\
Male, $n$ (\%) & $41(78.8)$ \\
Mean time from first IPF symptoms, years (SD) & $2.1(2.3)$ \\
Mean time from first IPF diagnosis, months (SD) & $0.9(1.1)$ \\
FVC\% predicted $\geq 80 \%, n(\%)$ & $22(42.3)$ \\
Mean DLco \% predicted (SD) & $51.7(13.5)$ \\
Comorbidities, $n$ (\%) & \\
At least one & $44(84.6)$ \\
Arterial hypertension & $30(57.7)$ \\
GERD & $15(28.8)$ \\
Diabetes mellitus & $14(26.9)$ \\
Benign prostatic hypertrophy & $7(13.5)$ \\
Hypercholesterolemia & $7(13.5)$ \\
Atherothrombotic disease* & $4(7.7)$ \\
Cerebrovascular disease & $3(5.8)$ \\
Anxiety/depression & $3(5.8)$ \\
Emphysema & $1(1.9)$ \\
Pulmonary hypertension & $1(1.9)$ \\
Lung cancer & $0(0.0)$ \\
Others & $19(36.5)$ \\
&
\end{tabular}

$\mathrm{DL}_{\mathrm{CO}}$, diffusing capacity of the lungs for carbon monoxide; $\mathrm{FVC}$ forced vital capacity; GERD, gastroesophageal reflux disease; IPF, idiopathic pulmonary fibrosis; SD, standard deviation. * Including coronary artery disease.

Table 2. FVC\% predicted of patients treated with nintedanib

\begin{tabular}{lll}
\hline & $N=52$ & $\begin{array}{l}\text { Mean FVC\% } \\
\text { predicted (SD) }\end{array}$ \\
\hline Baseline & 52 & $78.7(15.0)$ \\
3-month follow-up & 38 & $79.8(14.6)$ \\
6-month follow-up & 43 & $80.7(17.2)$ \\
9-month follow-up & 29 & $80.6(18.2)$ \\
12-month follow-up & 52 & $79.8(15.5)$ \\
\hline
\end{tabular}

FVC, forced vital capacity; SD, standard deviation.

For the 10 patients whose dose was reduced from 150 mg BID to $100 \mathrm{mg}$ BID, mean $( \pm \mathrm{SD}) \mathrm{FVC} \%$ predicted at baseline was $77.7 \%( \pm 20.0)$. There was no drop in mean FVC $\%$ predicted over time; mean $( \pm \mathrm{SD})$ values at followup were $83.6 \%( \pm 14.2 ; n=9), 80.7 \%( \pm 19.9 ; n=10), 82.3 \%$ $( \pm 27.5 ; n=4)$, and $81.0 \%( \pm 16.7 ; n=10)$ at $3,6,9$, and 12 months (Table 3). 
Table 3. FVC\% predicted of patients treated with $100 \mathrm{mg}$ nintedanib BID

\begin{tabular}{lll}
\hline & $N=10$ & Mean (SD) \\
\hline Baseline & 10 & $77.7(20.0)$ \\
3-month follow-up & 9 & $83.6(14.2)$ \\
6-month follow-up & 10 & $80.7(19.9)$ \\
9-month follow-up & 4 & $82.3(27.5)$ \\
12-month follow-up & 10 & $81.0(16.7)$ \\
\hline
\end{tabular}

BID, twice daily; FVC, forced vital capacity; SD, standard deviation.

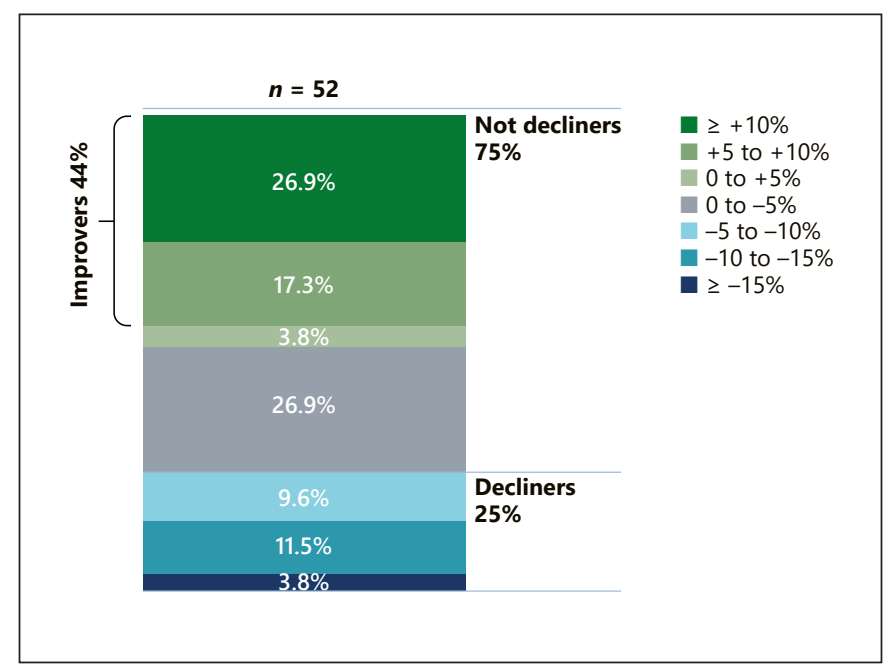

Fig. 1. Relative change in FVC\% predicted (baseline to 12-month follow-up) in patients treated with nintedanib. FVC, forced vital capacity.

\section{Anxiety and Depression}

At baseline, the mean $( \pm S D)$ total HADS score (anxiety and depression combined) was $11.5( \pm 6.9)(n=44)$. There was minimal variation in the HADS score over the observation period: mean $( \pm \mathrm{SD})$ HADS scores were 12.3 $( \pm 6.9)$ at 6 months $(n=36)$ and $10.8( \pm 8.7)$ at 12 months $(n=36)$, out of a total score of 42 (higher scores indicate greater levels of anxiety and depression).

\section{Proportion of Patients with Cough}

The proportion of patients with cough decreased from $50.0 \%(26 / 52)$ at baseline, $33.3 \%(16 / 48)$ at 3 months, $33.3 \%(16 / 48)$ at 6 months, $25.0 \%(11 / 44)$ at 9 months, to $21.2 \%(11 / 52)$ at 12 months. Based on the presence/absence of the symptom "cough" at the different study visits, the majority of patients had a variable pattern of cough that was present at some but not all visits (Table 4; online suppl. Table 1; for all online suppl. material, see www. karger.com/doi/10.1159/000521138).

\section{Exacerbations}

During the observation period, 2 patients (3.8\%) experienced at least one acute exacerbation. In total, four exacerbations occurred: two were classified as moderate and two were classified as severe, according to clinical judgment.

\section{AEs and SAEs}

In total, 27/52 patients (51.9\%) reported AEs between diagnosis and the end of observation (Table 5). The most common AE was diarrhea, occurring in 18/52 patients (34.6\%), followed by lack of appetite and weight loss, which were reported by 3 patients (5.8\%) each (Table 5). Ten patients received a reduced dose of nintedanib (100 mg BID) to manage AEs, and 2 patients discontinued nintedanib because of AEs: 1 patient discontinued due to diarrhea and nausea and another due to lack of appetite and weight loss. SAEs occurred in $2 / 52$ patients (3.8\%) between diagnosis and end of observation: 1 patient had angina and a myocardial infarction leading to death, and the other patient had respiratory insufficiency.

\section{Discussion}

In this post hoc analysis of the real-world FIBRONET study, the majority of patients with IPF receiving nintedanib for $\geq 7$ months were stable in terms of lung function during 12 months of observation. This finding is consistent with lung function data from the overall FIBRONET cohort, in which the mean FVC\% predicted was $80.0 \%$ at baseline ( $n=196)$ and $82.2 \%$ at 12 months $(n=133)$.

Our results are consistent with those of other realworld studies of nintedanib in IPF, in which treatment with nintedanib is associated with stabilization of lung function over 12 months of observation [5, 19]. After 12 months, a high proportion of patients in our study (75\%) were classified as stable or improving following nintedanib treatment. In a real-world study in Greece, FVC\% predicted was stable over 3 years in patients with IPF who received nintedanib; it was $73.3 \%$ at the start of treatment and $78.0 \%$ after 3 years of treatment, though the authors note that patients who attended follow-up visits may have had more stable disease than those who were lost to follow-up [5]. These results are similar to our findings, although the population in the Greek study had somewhat 
Table 4. Patterns of coughing across the study visits

\begin{tabular}{ll}
\hline Presence of cough & $N(\%)$ \\
\hline At baseline and every other visit & $4(7.7)$ \\
Not at baseline or any other visit & $15(28.8)$ \\
Variable (present at some visits, absent at some visits) & $20(38.5)$ \\
Remitting (present at $\geq 1$ visit followed by absence at $\geq 1$ visit) & $13(25.0)$
\end{tabular}

Table 5. Proportion of patients with AEs and SAEs during the observation period

\begin{tabular}{ll}
\hline & $N(\%)$ \\
& $N=52$ \\
\hline Any AE & $27(51.9)$ \\
Diarrhea & $18(34.6)$ \\
Lack of appetite & $3(5.8)$ \\
Weight loss & $3(5.8)$ \\
Bronchitis & $2(3.8)$ \\
Nausea & $2(3.8)$ \\
Increase of liver enzymes & $2(3.8)$ \\
Abdomen spasm & $1(1.9)$ \\
Abdominal pain & $1(1.9)$ \\
Chest pain & $1(1.9)$ \\
Dizziness & $1(1.9)$ \\
Respiratory tract infection & $1(1.9)$ \\
Stomach ache & $1(1.9)$ \\
Vomiting & $1(1.9)$ \\
Any SAE & $2(3.8)$ \\
\multicolumn{1}{c}{ Angina } & $1(1.9)$ \\
\multicolumn{1}{l}{ Myocardial infarction } & $1(1.9)$ \\
Respiratory insufficiency & $1(1.9)$ \\
\hline
\end{tabular}

$A E$, adverse event; $S A E$, serious adverse event.

more advanced disease at baseline, with almost half of patients having $\mathrm{FVC} \leq 50 \%$ predicted or $\mathrm{DL}_{\mathrm{CO}} \leq 35 \%$ predicted. An analysis of patients at three centers in the UK found that more patients had stable or improved lung function after treatment with nintedanib than in the period before they started nintedanib [20]. Similarly, in a cohort in Germany, nintedanib use was associated with stabilization of FVC, where $63 \%$ were classified as stable $(<5 \%$ decline in FVC) and $37 \%$ were classified as having disease progression ( $\geq 5 \%$ decline in FVC) at 6 months [18]. In another study, $62 \%$ of patients were considered stable after 9 months of follow-up [19]. One study of nintedanib for the treatment of severe IPF in Italy found that nintedanib stabilized $\mathrm{DL}_{\mathrm{CO}}$, but FVC decline was similar before and after nintedanib initiation during 6 months of treatment [10]. These real-world findings provide additional evidence on the effectiveness of nintedanib beyond the efficacy data in the INPULSIS randomized controlled trials. In these trials, there was a decline in FVC in the placebo arm of approximately $6 \%$ predicted and in the nintedanib arm of approximately $3 \%$ predicted over 52 weeks [3]. Differences in methodology and patient populations may account for the differences between realworld rates of FVC decline and those observed under strict clinical trial conditions. In our analysis, 10 out of the 52 patients had a dose reduction of nintedanib from $150 \mathrm{mg}$ BID to $100 \mathrm{mg}$ BID to manage AEs, similar to what was done in the INPULSIS studies, and lung function remained stable in these patients at 12 months without treatment interruption. Similar to previous realworld studies [18-20], we showed a high proportion of patients with stable $\mathrm{FVC} \%$ predicted and a group of patients who improved.

We also looked at cough and anxiety/depression in this analysis, as these are distressing aspects of IPF that can have a major impact on patients' lives [21, 22]. Anxiety and depression are common comorbidities in patients with IPF $[23,24]$ and can persist over a 12-month period. Reports from other registries have shown that this can correlate with cough and worsening dyspnea [25]. The overall proportion of patients reporting coughing decreased during the study; a quarter of patients had a remitting pattern of coughing where cough was present at one or more visits, followed by one or more subsequent visits where there was no cough. This suggests that this symptom may have a variable course over time. Low levels of anxiety and depression were observed (11.5 at baseline, out of a maximum score of 42.0 on the HADS), with minimal variation observed during the 12 months of observation.

Observational studies provide a valuable opportunity to examine the tolerability of drugs outside of a formal clinical trial setting. In line with previous clinical and real-world studies of nintedanib in IPF, diarrhea was the most common AE, occurring in more than a third of patients. The proportion of patients with diarrhea in this study is similar to other real-world studies of antifibrotics in IPF (ranging from $45.0 \%$ to $62.9 \%[5,18,20,26]$ ). SAEs occurred in 2 patients, and only 2 patients discontinued nintedanib after experiencing an AE. This discontinua- 
tion rate due to AEs is lower than that observed in pivotal phase III trials of nintedanib in IPF $(21.0 \%$ in INPULSIS- 1 and $17.6 \%$ in INPULSIS-2) [3]. The discontinuation rate is also toward the lower end of the range of discontinuation rates due to AEs observed in other realworld studies with a similar follow-up length, which range from $3.0 \%$ to $26.3 \%$ for nintedanib $[3,5,18,20,26]$.

One potential limitation of the study is that patients with less stable disease may have been less able to attend follow-up visits and perform lung function tests; although we imputed missing data, there is still a possibility that this may affect the results. Another limitation is that this is a specifically selected population of patients with at least 7 months of treatment with nintedanib. Eight patients who received nintedanib were excluded due to treatment discontinuation, switch, or interruption. This selection bias resulted in lower reported rates of discontinuation as patients who discontinued after less than 7 months were excluded. Finally, this was a post hoc and a descriptive analysis, so formal statistical testing was not performed, and the study was not designed to detect differences in outcomes between baseline and 12 months in the nintedanib subgroup.

\section{Conclusions}

This post hoc analysis of patients with IPF in Italy in the FIBRONET study adds to the body of evidence showing the effectiveness of nintedanib in the real world. Overall, our results show a high proportion of patients with stable disease over the 12 months of observation (i.e., no disease progression), a safety profile consistent with what is already known about the tolerability of nintedanib, and a low rate of discontinuation. The proportion of patients with cough also decreased. These findings are consistent with the efficacy and safety of nintedanib that has been demonstrated in controlled clinical trials.

\section{Acknowledgments}

Claire Scofield, MRes, of MediTech Media provided writing, editorial support, and formatting assistance, which was contracted and funded by Boehringer Ingelheim (BI). BI was given the opportunity to review the manuscript for medical and scientific accuracy, as well as intellectual property considerations. The FIBRONET study group includes a total of 20 members:

- Prof. Carlo Vancheri, Regional Referral Center for Rare Lung Diseases, University-Hospital "Policlinico G. Rodolico," Department of Clinical and Experimental Medicine, University of Catania, Catania, Italy
- Prof.ssa Sara Tomassetti, Department of Experimental and Clinical Medicine, Careggi University Hospital, Firenze, Italy

- Prof. Sergio Harari, Department of Clinical Sciences and Community Health, University of Milan; U.O. di Pneumologia e Terapia Semi-Intensiva Respiratoria, Ospedale San Giuseppe, IRCCS MultiMedica, Milan, Italy

- Prof. Alberto Pesci, Respiratory Unit, Department of Medicine, University of Milano-Bicocca, ASST Monza, Monza, Italy

- Prof. Carlo Albera, S.C. Pneumologia U., A.O.U. Città della Scienza e della Salute (Molinette), University of Turin, Torino, Italy

- Prof.ssa Paola Rottoli, U.O.C. di Pneumologia Universitaria, A.O.U. Senese Le Scotte, Siena, Italy

- Prof.ssa Marialuisa Bocchino, Clinica Malattie dell'Apparato Respiratorio, Università Federico II, Napoli, Italy

- Prof.ssa Anna Agnese Stanziola, Clinica Malattie dell'Apparato Respiratorio, Università Federico II, Napoli, Italy

- Prof. Fabrizio Luppi, U.O. di Clinica Pneumologica, Azienda Ospedaliera San Gerardo, Monza, Italy

- Dr. Alfredo Sebastiani, U.O.C. Pneumologia e Infettivologia Respiratoria, A.O. San Camillo Forlanini, Roma, Italy

- Prof. Donato Lacedonia, Pneumologia 4, Ospedale Colonnello D’Avanzo, Foggia, Italy

- Prof. Patrizio Vitulo, Servizio di Pneumologia, ISMETT di Palermo, Palermo, Italy

- Dr.ssa Laura Tavanti, U.O. di Pneumologia A.O.U. Pisana, Pisa, Italy

- Prof. Andrea Vianello, A.O.U. di Padova, Dip. Immunologia Clinica ed Ematologia, University-Hospital, Padova, Italy

- Prof.ssa Marina Saetta, Pneumologia Clinica, University-Hospital, Padova, Italy

- Dr. Stefano Marinari, U.O.C. di Pneumologia, Ospedale Clinicizzato Santissima Annunziata, Chieti, Italy

- Prof. Pietro Pirina, Pneumotisiologia Clinica Pneumologica, Policlinico Universitario di Sassari, Sassari, Italy

- Prof. Salvatore Valente, Unità complessa di Pneumologia, Policlinico Gemelli, Roma, Italy

- Dr. Tiberio Oggionni, Pneumologia, Policlinico San Matteo, Pavia, Italy

- Prof. Stefano Gasparini, Dip. Pneumologia, Ospedali Riuniti di Ancona, Ancona, Italy

\section{Statement of Ethics}

The protocol for the FIBRONET study was approved in July 2015 by an institutional review board/ethics committee (107/2015/ PO del registry EC), and written informed consent was obtained for all patients.

\section{Conflict of Interest Statement}

S.H. has acted as a clinical trial investigator, participated in scientific advisory boards, and delivered lectures for Boehringer Ingelheim, Roche, and Actelion. He has also received research grants from Boehringer Ingelheim. A.P. has received personal (speaker/ advisory board) fees from Boehringer Ingelheim and Roche, and his research activity is partially supported by project Premia. C. 
Albera has acted as a clinical trial investigator for Boehringer Ingelheim and Roche, participated in scientific advisory boards for Boehringer Ingelheim, Roche, MSD, Fibrogen, and GSK, and received research grants from Boehringer Ingelheim and Roche. V.P. has received personal fees from Boehringer Ingelheim. C. Amici is an employee of MediNeos Observational Research (Modena, Italy). G.C. is an employee of Boehringer Ingelheim (Italy). B.C. was an employee of Boehringer Ingelheim (Italy) at the time of this study. C.V. has received research grants and personal fees from Boehringer Ingelheim and Roche.

\section{Funding Sources}

The FIBRONET study was supported and funded by Boehringer Ingelheim Italia S.p.A. The authors did not receive payment related to the development of the manuscript.

\section{Author Contributions}

The authors meet criteria for authorship as recommended by the International Committee of Medical Journal Editors (ICMJE). The authors were fully responsible for all content and editorial decisions, had access to all data, were involved in all stages of development, and have approved the final version.

\section{Data Availability Statement}

To ensure independent interpretation of clinical study results, Boehringer Ingelheim grants all external authors access to relevant material, including participant-level clinical study data, as needed by them to fulfill their role and obligations as authors under the ICMJE criteria. Clinical study documents and participant clinical study data are available to be shared on request after publication of the primary manuscript in a peer-reviewed journal, and if regulatory activities are complete and other criteria met as per the BI Policy on Transparency and Publication of Clinical Study Data (see https://www.mystudywindow.com/msw/datasharing). Bona fide, qualified scientific and medical researchers are eligible to request access to the clinical study data with corresponding documentation describing the structure and content of the datasets. Upon approval, and governed by a Legal Agreement, data are shared in a secured data-access system for a limited period of 1 year, which may be extended upon request. Prior to providing access, clinical study documents and data will be examined, and, if necessary, redacted and de-identified, to protect the personal data of study participants and personnel, and to respect the boundaries of the informed consent of the study participants. Researchers should use the https://vivli.org/ link to request access to study data and visit https://www.mystudywindow.com/msw/datasharing for further information.

\section{References}

1 European Medicines Agency. Ofev (nintedanib): summary of product characteristics. 2015.

2 U.S. Food \& Drug Administration. OFEV (nintedanib): prescribing information. 2020.

3 Richeldi L, du Bois RM, Raghu G, Azuma A, Brown KK, Costabel U, et al. Efficacy and safety of nintedanib in idiopathic pulmonary fibrosis. N Engl J Med. 2014;370(22):2071-82.

4 Brown KK, Flaherty KR, Cottin V, Raghu G, Inoue $\mathrm{Y}$, Azuma A, et al. Lung function outcomes in the INPULSIS ${ }^{\star}$ trials of nintedanib in idiopathic pulmonary fibrosis. Respir Med. 2019;146:42-8.

5 Antoniou K, Markopoulou K, Tzouvelekis A, Trachalaki A, Vasarmidi E, Organtzis J, et al. Efficacy and safety of nintedanib in a Greek multicentre idiopathic pulmonary fibrosis registry: a retrospective, observational, cohort study. ERJ Open Res. 2020;6(1):00172-2019.

6 Behr J, Kreuter M, Hoeper MM, Wirtz H, Klotsche J, Koschel D, et al. Management of patients with idiopathic pulmonary fibrosis in clinical practice: the INSIGHTS-IPF registry. Eur Respir J. 2015;46(1):186-96.

7 Fernandez-Fabrellas E, Molina-Molina M, Soriano JB, Portal JAR, Ancochea J, Valenzuela C, et al. Demographic and clinical profile of idiopathic pulmonary fibrosis patients in Spain: the SEPAR National Registry. Respir Res. 2019;20(1):127.
8 Guenther A, Krauss E, Tello S, Wagner J, Paul B, Kuhn S, et al. The European IPF registry (eurIPFreg): baseline characteristics and survival of patients with idiopathic pulmonary fibrosis. Respir Res. 2018;19(1):141.

9 Vancheri C, Sebastiani A, Tomassetti S, Pesci A, Rogliani P, Tavanti L, et al. Pirfenidone in real life: a retrospective observational multicentre study in Italian patients with idiopathic pulmonary fibrosis. Respir Med. 2019;156: 78-84.

10 Harari S, Caminati A, Poletti V, Confalonieri M, Gasparini S, Lacedonia D, et al. A Real-life multicenter national study on nintedanib in severe idiopathic pulmonary fibrosis. Respiration. 2018;95(6):433-40.

11 Harari S, Caminati A, Albera C, Vancheri C, Poletti V, Pesci A, et al. Efficacy of pirfenidone for idiopathic pulmonary fibrosis: an Italian real life study. Respir Med. 2015; 109(7):904-13.

12 Mortimer KM, Bartels DB, Hartmann N, Capapey J, Yang J, Gately R, et al. Characterizing health outcomes in idiopathic pulmonary fibrosis using US health claims data. Respiration. 2020;99(2):108-18.

13 Jo HE, Glaspole I, Grainge C, Goh N, Hopkins PM, Moodley Y, et al. Baseline characteristics of idiopathic pulmonary fibrosis: analysis from the Australian Idiopathic Pulmonary Fibrosis Registry. Eur Respir J. 2017;49(2): 1601592.
14 Poletti V, Vancheri C, Albera C, Harari S, Pesci A, Rottoli P, et al. Clinical course of IPF Italian patients during 12-month of observation: results from the FIBRONET observational study. Respir Res. 2021;22(1):66.

15 Raghu G, Collard HR, Egan JJ, Martinez FJ, Behr J, Brown KK, et al. An official ATS/ERS/ JRS/ALAT statement: idiopathic pulmonary fibrosis: evidence-based guidelines for diagnosis and management. Am J Respir Crit Care Med. 2011;183(6):788-824.

16 Ponce MC, Sharma S. Pulmonary function tests. StatPearls [Internet]. Treasure Island, FL: StatPearls Publishing; 2021.

17 Juarez MM, Chan AL, Norris AG, Morrissey BM, Albertson TE. Acute exacerbation of idiopathic pulmonary fibrosis-a review of current and novel pharmacotherapies. J Thorac Dis. 2015;7(3):499-519.

18 Bonella F, Kreuter M, Hagmeyer L, Neurohr C, Keller C, Kohlhaeufl MJ, et al. Insights from the German compassionate use program of nintedanib for the treatment of idiopathic pulmonary fibrosis. Respiration. 2016; 92(2):98-106.

19 Brunnemer E, Walscher J, Tenenbaum S, Hausmanns J, Schulze K, Seiter M, et al. Realworld experience with nintedanib in patients with idiopathic pulmonary fibrosis. Respiration. 2018;95(5):301-9. 
20 Toellner H, Hughes G, Beswick W, Crooks MG, Donaldson C, Forrest I, et al. Early clinical experiences with nintedanib in three UK tertiary interstitial lung disease centres. Clin Transl Med. 2017;6(1):41.

21 van Manen MJ, Birring SS, Vancheri C, Cottin V, Renzoni EA, Russell AM, et al. Cough in idiopathic pulmonary fibrosis. Eur Respir Rev. 2016;25(141):278-86.

22 Lee YJ, Choi SM, Lee YJ, Cho YJ, Yoon HI, Lee $\mathrm{JH}$, et al. Clinical impact of depression and anxiety in patients with idiopathic pulmonary fibrosis. PLoS One. 2017;12(9):e0184300.
23 Ryerson CJ, Arean PA, Berkeley J, CarrieriKohlman VL, Pantilat SZ, Landefeld CS, et al. Depression is a common and chronic comorbidity in patients with interstitial lung disease. Respirology. 2012;17(3):525-32.

24 Lindell KO, Olshansky E, Song MK, Zullo TG Gibson KF, Kaminski N, et al. Impact of a disease-management program on symptom burden and health-related quality of life in patients with idiopathic pulmonary fibrosis and their care partners. Heart Lung. 2010;39(4):304-13.
25 Glaspole IN, Watson AL, Allan H, Chapman S, Cooper WA, Corte TJ, et al. Determinants and outcomes of prolonged anxiety and depression in idiopathic pulmonary fibrosis. Eur Respir J. 2017;50(2): 1700168.

26 Galli JA, Pandya A, Vega-Olivo M, Dass C, Zhao $\mathrm{H}$, Criner GJ. Pirfenidone and nintedanib for pulmonary fibrosis in clinical practice: tolerability and adverse drug reactions. Respirology. 2017;22(6):1171-8. 